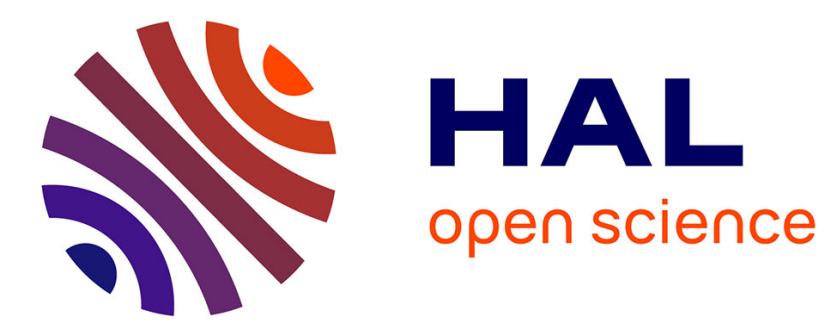

\title{
On Abel's Concept of Doubt and Pessimism
}

Elyès Jouini, Clotilde Napp

\section{To cite this version:}

Elyès Jouini, Clotilde Napp. On Abel's Concept of Doubt and Pessimism. Journal of Economic Dynamics and Control, 2008, 32, pp.3682-3694. halshs-00176611v2

\section{HAL Id: halshs-00176611 \\ https://shs.hal.science/halshs-00176611v2}

Submitted on 2 Jun 2010

HAL is a multi-disciplinary open access archive for the deposit and dissemination of scientific research documents, whether they are published or not. The documents may come from teaching and research institutions in France or abroad, or from public or private research centers.
L'archive ouverte pluridisciplinaire HAL, est destinée au dépôt et à la diffusion de documents scientifiques de niveau recherche, publiés ou non, émanant des établissements d'enseignement et de recherche français ou étrangers, des laboratoires publics ou privés. 


\title{
On Abel's concept of doubt and pessimism
}

\author{
E. Jouini \\ CEREMADE Université Paris 9-Dauphine \\ Fellow of the Institut universitaire de France \\ C. Napp \\ CEREMADE Université Paris 9-Dauphine and CREST
}

September 27, 2006 


\begin{abstract}
In this paper, we characterize subjective probability beliefs leading to a higher equilibrium market price of risk. We establish that Abel's result on the impact of doubt on the risk premium is not correct (see Abel, A., 2002. An exploration of the effects of pessimism and doubt on asset returns. Journal of Economic Dynamics and Control, 26, 1075-1092). We introduce, on the set of subjective probability beliefs, market-price-of-risk dominance concepts and we relate them to well known dominance concepts used for comparative statics in portfolio choice analysis. In particular, the necessary first order conditions on subjective probability beliefs in order to increase the market price of risk for all nondecreasing utility functions appear as equivalent to the monotone likelihood ratio property.

Keywords : pessimism, optimism, doubt, stochastic dominance, risk premium, market price of risk, riskiness, portfolio dominance, monotone likelihood ratio.
\end{abstract}

JEL numbers : D81, G11 


\section{Introduction}

We start from an equilibrium model in which the (representative) agent is endowed with a subjective probability $Q$ different from the initial probability $P$. We want to measure the impact of this subjective probability on the equilibrium market price of risk. In particular, in relation with the Equity premium puzzle ${ }^{1}$, we are interested in the characterization of the set of subjective probabilities leading to an increase of the market price of risk for all utility functions in a given class.

Starting from the empirical findings ${ }^{2}$ of Cecchetti et al. (2000), Abel (2002) has considered a closely related problem. He defines pessimism (resp.doubt) as a First Stochastic Dominance shift (resp. mean preserving spread) of the representative agent's subjective distribution of aggregate consumption. In order to analyze the impact of pessimism on the equilibrium risk premium, Abel (2002) introduces the stronger notion of uniform pessimism ${ }^{3}$ and proves, for a representative agent endowed with a power utility function, that uniform pessimism is a sufficient condition for an increase of the equilibrium risk premium. He also proves for power utility functions and using Taylor series expansions that doubt increases the equilibrium risk premium. However this result is wrong as shown on a simple couterexample provided in Section 4.

Our aim is to characterize subjective beliefs leading to a higher equilibrium market price of risk for large classes of utility functions. More precisely, according to which class of utility functions we consider, we define the market-price-of-risk dominance of the first-order (for all nondecreasing utility functions) and of the second-order (for all nondecreasing and concave utility functions).

In fact, changes in beliefs (probabilities) can be interpreted in terms of changes in risk (random variables) explored among others by Rothschild and Stiglitz (1970 a. and b.), Meyer and Ormiston (1983), Landsberger and Meilijson (1990, 1993), Hadar and Seo (1990), Ormiston and Schlee (1993), Gollier (1995), Gollier and Schlesinger (2002).

We show that our first-order (resp. second-order) market-price-of-risk dominance is equivalent, when restated in terms of random variables, to the monotone likelihood ratio dominance (resp. portfolio dominance) studied by Landsberger

\footnotetext{
${ }^{1}$ See Mehra and Prescott (1985) or Kocherlakota (1996) for a survey.

${ }^{2}$ The authors prove that a model in which consumers exhibit pessimistic beliefs can better match sample moments of asset returns than can a rational expectations model. See also the empirical study in Giordani and Söderlind (2003), where the authors provide evidence of pessimism in investors forecasts.

${ }^{3}$ defined by $Q(X \leq t)=P\left(X \leq e^{\Delta} t\right)$ for some $\Delta>0$.
} 
and Meilijson (1990, 1993) and Gollier (1997).

We use these characterizations to provide examples of first-order and secondorder market-price-of-risk dominance. In particular, we explore the case where the total wealth is normally or log-normally distributed.

\section{Market-price-of-risk dominance}

Let $(\Omega, \mathcal{F}, P)$ be a given probability space. We consider a standard 2 dates Lucasfruit tree economy, except that we allow the representative agent to have a subjective belief/opinion. The representative agent solves a standard utility maximization problem. He has a current income at date $t=0,1$ denoted by $\left(e_{0}, e\right)$ and a von Neumann-Morgenstern utility function for consumption of the form $u_{0}\left(c_{0}\right)+E^{Q}[u(c)]$, where $Q$ is a probability measure absolutely continuous with respect to $P$ which corresponds to the subjective belief of the agent. Throughout the paper $e$ is assumed to be nonnegative. If we denote by $M$ the nonnegative density of $Q$ with respect to $P$, then the utility function can be rewritten as $u_{0}\left(c_{0}\right)+E^{P}[M u(c)]$. We suppose that the economy is in equilibrium, i.e. that there exists a nonnegative, nonzero and uniformly bounded price process ${ }^{4}$ $q$ such that the optimal consumption plan for the representative agent (under his budget constraint) coincides with the aggregate wealth of the economy, i.e. $e=\arg \max _{E^{P}\left[\left(c_{0}-e_{0}\right)+q(c-e)\right] \leq 0}\left\{u_{0}\left(c_{0}\right)+E^{P}[M u(c)]\right\}$. Such an equilibrium, when it exists, can be characterized by the first order necessary conditions for individual optimality. When the equilibrium is interior, these conditions are given by $M u^{\prime}(e)=u_{0}^{\prime}\left(e_{0}\right) q$.

It is easy to obtain, as in the classical setting, the CCAPM formula. We suppose the existence of a riskless asset with price process $S^{0}$ such that $S_{0}^{0}=1$ and $S_{1}^{0}=\left(1+r^{f}\right)$ for some risk free rate $r^{f}$. We consider a risky asset with positive price process $S$ and associated rate of return between date 0 and 1 denoted by $R \equiv \frac{S_{1}}{S_{0}}-1$. In such a context, since $q S$ is a $P$-martingale, we obtain

$$
E^{P}[R]-r^{f}=-\operatorname{cov}^{P}\left[\frac{q}{E^{P}[q]}, R\right]
$$

or equivalently

$$
E^{P}[R]-r^{f}=-\operatorname{cov}^{P}\left[\frac{M u^{\prime}(e)}{E^{P}\left[M u^{\prime}(e)\right]}, R\right] .
$$

\footnotetext{
${ }^{4}$ Prices are in terms of date- 0 consumption units.
} 
The formula for the risk premium in the standard setting is given by

$$
E^{P}[R]-r^{f}=-\operatorname{cov}^{P}\left[\frac{u^{\prime}(e)}{E^{P}\left[u^{\prime}(e)\right]}, R\right] .
$$

Note that the quantity $-\operatorname{cov}^{P}\left[\frac{M u^{\prime}(e)}{E^{P}\left[M u^{\prime}(e)\right]}, R\right]$ that corresponds to the risk premium in the subjective beliefs setting is not equal to $-\operatorname{cov}^{Q}\left[\frac{u^{\prime}(e)}{E^{Q}\left[u^{\prime}(e)\right]}, R\right]$ which would correspond to the subjective risk premium $E^{Q}[R]-r^{f}$. Indeed, our aim is to compare the risk premia from the econometrician point of view, i.e. under the same "objective" probability $P$. The risk premia we consider correspond then to average realized risk premia and not to ex-ante subjectively expected ones.

In order to analyze the impact of the subjective belief on the value of the risk premium, we must take into account the fact that the possible returns $R$ obtained in equilibrium are not the same in both settings. What exactly is to be compared ?

We consider an asset, whose terminal payoff at date 1 is given by the aggregate wealth $e$. Notice that such an asset exists by market completeness, but obviously does not have the same price and return in both settings. However, the "risk level" of such an asset ${ }^{5}$ is modified in the same proportions as the returns. We propose to compare the market price of risk (i.e., the ratio between the risk premium and the "level of risk") in both settings, which leads us to compare $-\operatorname{cov}^{P}\left[\frac{u^{\prime}(e)}{E^{P}\left[u^{\prime}(e)\right]}, e\right]$ with $-\operatorname{cov}^{P}\left[\frac{M u^{\prime}(e)}{E^{P}\left[M u^{\prime}(e)\right]}, e\right]$. Hence, the subjective belief setting leads to a higher market price of risk if and only if

$$
\frac{E^{Q}\left[u^{\prime}(e) e\right]}{E^{Q}\left[u^{\prime}(e)\right]} \leq \frac{E^{P}\left[u^{\prime}(e) e\right]}{E^{P}\left[u^{\prime}(e)\right]} .
$$

We wish to characterize the set of such probabilities. Note that the probability measures for which the market price of risk increases are those for which the equilibrium relative price (of $e$ ) decreases and they are also those for which the equilibrium risk premium in the sense of Abel (2002) increases.

Indeed, in the standard model, the equilibrium price $p^{P}(e)$ for $e$ is given by $E^{P}[q e]=E^{P}\left[u^{\prime}(e) e\right] / u_{0}^{\prime}\left(e_{0}\right)$ and the price $p^{P}(1)$ for a riskless asset whose payoff is always 1 is given by $E^{P}[q]=E^{P}\left[u^{\prime}(e)\right] / u_{0}^{\prime}\left(e_{0}\right)$, hence the equilibrium relative price $r p^{P}(e)$ is given by $\frac{E^{P}\left[u^{\prime}(e) e\right]}{E^{P}\left[u^{\prime}(e)\right]}$, which is then to be compared to the equilibrium relative price in the subjective beliefs setting $r p^{Q}(e)=\frac{E^{Q}\left[u^{\prime}(e) e\right]}{E^{Q}\left[u^{\prime}(e)\right]}$. Besides, in Abel

\footnotetext{
${ }^{5}$ Measured either by the standard deviation or by a market (resp. consumption) beta.
} 
(2002), the risk premium is defined as the ratio between the expected return of the considered asset and the riskfree return, i.e.

$$
\frac{E^{P}[R+1]}{1+r^{f}}=\frac{E^{P}\left[\frac{e}{p^{P}(e)}\right]}{1+r^{f}}=E^{P}[e] \frac{1 /\left(1+r^{f}\right)}{p^{P}(e)}=\frac{E^{P}[e]}{r p^{P}(e)},
$$

so that the equilibrium risk premium in the subjective beliefs setting is higher than in the standard setting if and only if (2.3).

We introduce the following definitions. We shall consider the sets $\mathcal{U}_{1}$ of all continuous nondecreasing functions $u$ on $\mathbb{R}_{+}^{*}$ such that $\limsup _{x \rightarrow \infty} u^{\prime}(x)<\infty$ and $\mathcal{U}_{2}$ of all continuous nondecreasing concave ${ }^{6}$ functions $u$ on $\mathbb{R}_{+}^{*}$.

Definition 2.1. Let e be a nonnegative random variable on $\Omega$ and let $F$ be the sigma-algebra generated by e. Let $Q_{1}$ and $Q_{2}$ denote two probability measures on $(\Omega, F)$ such that $E^{Q_{i}}[e]<\infty, i=1,2$. We say that $Q_{1}$ dominates $Q_{2}$ - with respect to $e$ - in the sense of the market price of risk of order $i\left(Q_{1} \succcurlyeq_{M P R_{i}} Q_{2}\right)$ when for all utility functions $u$ in $\mathcal{U}_{i}, E^{Q_{1}}\left[u^{\prime}(e) e\right] E^{Q_{2}}\left[u^{\prime}(e)\right] \leq E^{Q_{2}}\left[u^{\prime}(e) e\right] E^{Q_{1}}\left[u^{\prime}(e)\right]$.

Note that we do not refer anymore to an objective probability in this definition. However it suffices to consider any probability measure $P$ such that $Q_{1}$ and $Q_{2}$ are absolutely continuous with respect to $P$ to recover the interpretation of $\frac{E^{Q_{1}}\left[u^{\prime}(e) e\right]}{E^{Q_{1}}\left[u^{\prime}(e)\right]}$ (resp. $\left.\frac{E^{Q_{2}}\left[u^{\prime}(e) e\right]}{E^{Q_{2}}\left[u^{\prime}(e)\right]}\right)$ as the equilibrium relative price of $e$ in a market where the representative agent subjective belief is given by $Q_{1}$ (resp. by $Q_{2}$ ). Such a probability $P$ can be, for instance, any positive combination of $Q_{1}$ and $Q_{2}$.

Remark also that for $u$ in $\mathcal{U}_{i}$ such that $Q_{i}\left(u^{\prime}(e) \neq 0\right) \neq 0, i=1,2$ we have $\lim \sup _{x \rightarrow \infty} u^{\prime}(x)<\infty$ and since $e$ is integrable, $\frac{E^{Q_{i}}\left[u^{\prime}(e) e 1_{e \geq x}\right]}{E^{Q_{i}}\left[u^{\prime}(e) 1_{e \geq x}\right]}$ is well defined for $x$ sufficiently large. Furthermore this quantity decreases when $x$ goes to zero. Hence $\frac{E^{Q_{i}\left[u^{\prime}(e) e\right]}}{E^{Q_{i}}\left[u^{\prime}(e)\right]}$ is well defined at least as the limit of $\frac{E^{Q_{i}}\left[u^{\prime}(e) e 1_{e \geq x}\right]}{E^{Q_{i}}\left[u^{\prime}(e) 1_{e \geq x}\right]}$ when $x$ goes to zero.

Sections 3 and 4 are respectively devoted to first-order and second-order marketprice-of-risk dominance.

\section{First-order market-price-of-risk dominance}

We first show with a counter-example that Abel's concept of pessimism defined through First Stochastic Dominance is not sufficient to ensure $\mathrm{MPR}_{1}$ dominance.

\footnotetext{
${ }^{6}$ Note that all functions in $\mathcal{U}_{1}$ are almost everywhere differentiable and all functions in $\mathcal{U}_{2}$ are almost everywhere twice differentiable.
} 
We then prove the equivalence between $M P R_{1}$ dominance and Monotone Likelihood Ratio dominance, introduced by Landsberger and Meilijson (1990) and finally use this characterization to provide examples and interpret the $\mathrm{MPR}_{1}$ dominance.

Abel (2002) uses the term pessimism to mean that the representative agent's subjective distribution of aggregate consumption is first-order stochastically dominated by the objective distribution. It has long been shown in the literature that the standard stochastic orders are not sufficient in portfolio settings; in particular, a first stochastic dominance improvement of the returns of the risky asset needs not generate a higher demand for it (see e.g. Fishburn and Porter, 1976 or Hadar and Seo, 1990). As a consequence, it is intuititive that a FSD shift, as in Abel (2002), should not necessarily reduce the equilibrium relative price of the risky asset. We show it on the following simple example.

Suppose that $e \sim \mathcal{U}_{[1 ; 3]}$ and that the density of $Q$ with respect to $P$ is given by $f(e)$ where

$$
f(x)=\left\{\begin{array}{cc}
x & \text { on }[1 ; 2[ \\
x-2 & \text { on }[2 ; 3]
\end{array} .\right.
$$

It is easy to see on Figure 1 that $P$ dominates $Q$ in the sense of the first stochastic dominance. The probability measure $Q$ is then pessimistic in the sense of Abel (2002).

If the representative agent utility function is such that $u^{\prime}(x)=1_{x \leq 2}$, we can easily check that $\operatorname{cov}^{P_{u}}(e, f(e))>0$ where $P_{u}$ has a density $\frac{u^{\prime}(e)}{E^{P}\left[u^{\prime}(e)\right]}$ with respect to $P$. Indeed, $P_{u}$ does not charge $\left.] 2 ; 3\right]$ and $f(e)$ increases with $e$ under $P_{u}$. Now, since $\left(\frac{E^{Q}\left[u^{\prime}(e) e\right]}{E^{Q}\left[u^{\prime}(e)\right]}-\frac{E^{P}\left[u^{\prime}(e) e\right]}{E^{P}\left[u^{\prime}(e)\right]}\right)=\frac{E^{P}\left[u^{\prime}(e)\right]}{E^{Q}\left[u^{\prime}(e)\right]} \operatorname{cov}^{P_{u}}(e, f(e))$, this leads to a decrease of the equilibrium risk premium.

As it can be seen in Figure 2., $Q$ is "pessimistic" with respect to $P$ but "optimistic" with respect to $P_{u}$ (since it is almost surely increasing under $P_{u}$ ) and this is the reason why we obtain a decrease of the equilibrium risk premium.

One could argue that this representative agent's utility function is too specific since it is not strictly increasing nor strictly concave and that $P_{u}$ is not equivalent to $P$. However, small perturbations of this function would lead to the same result. Furthermore, we can check that we would obtain the same result for well chosen power utility functions (e.g. $u^{\prime}(x)=x^{-6}$ ). Hence, even in the class of utility functions studied in Abel (2002), the concept of pessimism introduced therein is not sufficient to guarantee an increase of the equilibrium risk premium.

Notice that Abel (2002) does not claim that FSD is sufficient to ensure market- 
price-of-risk dominance for power utility functions. He does not discuss this point; he introduces a stronger concept of pessimism, called uniform pessimism ${ }^{7}$, and proves that this stronger notion is sufficient.

The next Proposition characterizes the first-order market-price-of-risk dominance. We recall the definition of the monotone likelihood ratio order, widely used in the statistical literature and first introduced in the context of portfolio problems by Landsberger and Meilijson $(1990)^{8}$. A distribution $G$ is said to dominate a distribution $F$ in the sense of the MLR order $\left(G \succcurlyeq_{M L R} F\right)$ if there exist numbers $-\infty \leq x_{1} \leq x_{2} \leq \infty$ and a nondecreasing function $h:\left[x_{1}, x_{2}\right] \rightarrow[0, \infty]$ such that $G(C)=0, \forall C<x_{1}, F(D)=1, \forall D>x_{2}$, and $d G(x)=h(x) d F(x)$ on $\left[x_{1}, x_{2}\right]$.

Proposition 3.1. Let $Q_{1}$ and $Q_{2}$ denote two probability measures on $(\Omega, F)$ and let $F_{1}$ (resp. $F_{2}$ ) denote the distribution of e under $Q_{1}$ (resp. $\left.Q_{2}\right)$. Then $Q_{1} \succcurlyeq_{M P R_{1}} Q_{2}$ if and only if $F_{2} \succcurlyeq_{M L R} F_{1}$.

Proof. 1. Let us assume that $Q_{1}$ and $Q_{2}$ are equivalent and that $Q_{1} \succcurlyeq_{M P R_{1}} Q_{2}$. We have then $\frac{E^{Q_{1}}\left[u^{\prime}(e) e\right]}{E^{Q_{1}}\left[u^{\prime}(e)\right]} \leq \frac{E^{Q_{2}}\left[u^{\prime}(e) e\right]}{E^{Q_{2}}\left[u^{\prime}(e)\right]}$ for any $u$ in $\mathcal{U}_{1}$. Since $F$ is generated by $e, \frac{d Q_{2}}{d Q_{1}}$ can be written in the form $\frac{d Q_{2}}{d Q_{1}}=h(e)$. Let us prove that the function $h$ is nondecreasing, or more precisely that the random variable $\varphi$ defined on $\left(\mathbb{R}^{2}, \mathcal{B}\left(\mathbb{R}^{2}\right)\right)$ by $\varphi\left(x_{1}, x_{2}\right)=h\left(x_{2}\right)-h\left(x_{1}\right)$ is $P_{e} \otimes P_{e}$ almost surely nonnegative on the open half plane $\left\{\left(x_{1}, x_{2}\right), x_{1}<x_{2}\right\}$.

Let us assume on the contrary that there exist two Borel real sets $A_{1}$ and $A_{2}$ such that ${ }^{9} A_{1}<A_{2}, P_{e}\left(A_{i}\right) \neq 0, i=1,2$ and $\varphi$ is $P_{e} \otimes P_{e}$ almost surely negative on $A_{1} \times A_{2}$. We can choose $A_{1}$ and $A_{2}$ such that $h\left(A_{1}\right) \subset[a, a+\varepsilon]$ and $h\left(A_{2}\right) \subset[b-\varepsilon, b]$ for some $a>b$ and $0<\varepsilon<a-b$.

We have then $\frac{Q_{2}\left(e \in A_{1}\right)}{Q_{1}\left(e \in A_{1}\right)}=\frac{E^{Q_{1}}\left[\frac{d Q_{2}}{d Q_{1}} 1_{e \in A_{1}}\right]}{Q_{1}\left(e \in A_{1}\right)} \in[a, a+\varepsilon]$ and $\frac{Q_{2}\left(e \in A_{2}\right)}{Q_{1}\left(e \in A_{2}\right)} \in[b-\varepsilon, b]$. Let $\alpha>0$ be given and let $\theta=\frac{Q_{1}\left(A_{1}\right)}{Q_{1}\left(A_{1}\right)+\alpha Q_{1}\left(A_{2}\right)}$. We have

$$
\theta a+(1-\theta)(b-\varepsilon) \leq \theta \frac{Q_{2}\left(A_{1}\right)}{Q_{1}\left(A_{1}\right)}+(1-\theta) \frac{Q_{2}\left(A_{2}\right)}{Q_{1}\left(A_{2}\right)} \leq \theta(a+\varepsilon)+(1-\theta) b
$$

\footnotetext{
${ }^{7}$ defined by $Q(X \leq t)=P\left(X \leq e^{\Delta} t\right)$ for some $\Delta>0$.

${ }^{8}$ More precisely, Landsberger and Meilijson (1990) showed that in the standard portfolio problem a MLR shift in the distribution of returns of the risky asset leads to an increase in demand for the risky asset for all agents with nonincreasing utilities.

${ }^{9}$ For two real subsets $A_{1}$ and $A_{2}, A_{1}<A_{2}$ stands for $\forall\left(a_{1}, a_{2}\right) \in A_{1} \times A_{2}, a_{1}<a_{2}$.
} 
and for $\alpha \in\left(\frac{Q_{1}\left(A_{1}\right)}{Q_{1}\left(A_{2}\right)} \frac{\varepsilon}{a-b}, \frac{Q_{1}\left(A_{1}\right)}{Q_{1}\left(A_{2}\right)} \frac{a-b}{\varepsilon}\right)$, we obtain $\theta \frac{Q_{2}\left(A_{1}\right)}{Q_{1}\left(A_{1}\right)}+(1-\theta) \frac{Q_{2}\left(A_{2}\right)}{Q_{1}\left(A_{2}\right)} \in(b, a)$. It follows then that for such $\alpha, \frac{d Q_{2}}{d Q_{1}}$ is higher (resp. lower) than $\frac{Q_{2}\left(A_{1}\right)+\alpha Q_{2}\left(A_{2}\right)}{Q_{1}\left(A_{1}\right)+\alpha Q_{1}\left(A_{2}\right)}$ on $A_{1}$ (resp. $\left.A_{2}\right)$.

Let $\gamma$ denote some real number in $\left[\sup A_{1}, \inf A_{2}\right]$ and let the utility function $u$ be defined by $u^{\prime}(x)=1_{A_{1}}+\alpha 1_{A_{2}}$. We have

$$
\begin{aligned}
\frac{E^{Q_{2}}\left[e u^{\prime}(e)\right]}{E^{Q_{2}}\left[u^{\prime}(e)\right]} & =\frac{E^{Q_{2}}\left[(e-\gamma) 1_{A_{1}}\right]+\alpha E^{Q_{2}}\left[(e-\gamma) 1_{A_{2}}\right]}{E^{Q_{2}}\left[1_{A_{1}}\right]+\alpha E^{Q_{2}}\left[1_{A_{2}}\right]}+\gamma \\
& =\frac{E^{Q_{1}}\left[(e-\gamma) \frac{d Q_{2}}{d Q_{1}} 1_{A_{1}}\right]+\alpha E^{Q_{1}}\left[(e-\gamma) \frac{d Q_{2}}{d Q_{1}} 1_{A_{2}}\right]}{E^{Q_{2}}\left[1_{A_{1}}\right]+\alpha E^{Q_{2}}\left[1_{A_{2}}\right]}+\gamma .
\end{aligned}
$$

Now, since $(e-\gamma) \leq 0$ and $\frac{d Q_{2}}{d Q_{1}}>\frac{Q_{2}\left(A_{1}\right)+\alpha Q_{2}\left(A_{2}\right)}{Q_{1}\left(A_{1}\right)+\alpha Q_{1}\left(A_{2}\right)}$ on $A_{1}$, we have $(e-\gamma) \frac{d Q_{2}}{d Q_{1}} \leq$ $(e-\gamma) \frac{Q_{2}\left(A_{1}\right)+\alpha Q_{2}\left(A_{2}\right)}{Q_{1}\left(A_{1}\right)+\alpha Q_{1}\left(A_{2}\right)}$ on $A_{1}$. Analogously we get that $(e-\gamma) \frac{d Q_{2}}{d Q_{1}} \leq \frac{Q_{2}\left(A_{1}\right)+\alpha Q_{2}\left(A_{2}\right)}{Q_{1}\left(A_{1}\right)+\alpha Q_{1}\left(A_{2}\right)}(e-\gamma)$ on $A_{2}$ and one of these inequalities is strict. Hence

$$
\begin{aligned}
\frac{E^{Q_{2}}\left[e u^{\prime}(e)\right]}{E^{Q_{2}}\left[u^{\prime}(e)\right]} & <\frac{Q_{2}\left(A_{1}\right)+\alpha Q_{2}\left(A_{2}\right)}{Q_{1}\left(A_{1}\right)+\alpha Q_{1}\left(A_{2}\right)} \frac{E^{Q_{1}}\left[(e-\gamma) 1_{A_{1}}\right]+\alpha E^{Q_{1}}\left[(e-\gamma) 1_{A_{2}}\right]}{E^{Q_{2}}\left[1_{A_{1}}\right]+\alpha E^{Q_{2}}\left[1_{A_{2}}\right]}+\gamma \\
& <\frac{E^{Q_{1}}\left[(e-\gamma) 1_{A_{1}}\right]+\alpha E^{Q_{1}}\left[(e-\gamma) 1_{A_{2}}\right]}{E^{Q_{1}}\left[1_{A_{1}}\right]+\alpha E^{Q_{1}}\left[1_{A_{2}}\right]}+\gamma \\
& <\frac{E^{Q_{1}}\left[e u^{\prime}(e)\right]}{E^{Q_{1}}\left[u^{\prime}(e)\right]}
\end{aligned}
$$

which contradicts the fact that $Q_{1} \succcurlyeq_{M P R_{1}} Q_{2}$.

Conversely, $\frac{E^{Q_{2}}\left[u^{\prime}(e) e\right]}{E^{Q_{2}}\left[u^{\prime}(e)\right]}=\frac{E^{Q_{1}}\left[\frac{d Q_{2}}{d Q_{1}} u^{\prime}(e) e\right]}{E^{Q_{1}}\left[\frac{d Q_{2}}{d Q_{1}} u^{\prime}(e)\right]}=\frac{E^{Q_{u}}\left[\frac{d Q_{2}}{d Q_{1}} e\right] E^{Q_{1}}\left[u^{\prime}(e)\right]}{E^{Q_{1}}\left[\frac{d Q_{2}}{d Q_{1}} u^{\prime}(e)\right]}$ where $Q_{u}$ is defined by a density with respect to $Q_{1}$ equal (up to a constant) to $u^{\prime}(e)$. Since $\frac{d Q_{2}}{d Q_{1}}$ is nondecreasing in $e$, we have

$$
E^{Q_{u}}\left[\frac{d Q_{2}}{d Q_{1}} e\right] \geq E^{Q_{u}}\left[\frac{d Q_{2}}{d Q_{1}}\right] E^{Q_{u}}[e]
$$

hence

$$
\begin{aligned}
\frac{E^{Q_{2}}\left[u^{\prime}(e) e\right]}{E^{Q_{2}}\left[u^{\prime}(e)\right]} & \geq \frac{E^{Q_{u}}\left[\frac{d Q_{2}}{d Q_{1}}\right] E^{Q_{u}}[e] E^{Q_{1}}\left[u^{\prime}(e)\right]}{E^{Q_{1}}\left[\frac{d Q_{2}}{d Q_{1}} u^{\prime}(e)\right]} \\
& \geq \frac{E^{Q_{1}}\left[u^{\prime}(e) e\right]}{E^{Q_{1}}\left[u^{\prime}(e)\right]} .
\end{aligned}
$$


2. Let us now consider the general case. We first prove that if $Q_{1} \succcurlyeq_{M P R_{1}} Q_{2}$, subsets on which $Q_{1}$ is zero correspond to values of $e$ at the right-end of the real line. Let $\left(A_{i}\right)_{i=1,2}$ be disjoint real subsets such that ${ }^{10} \operatorname{co}\left(A_{1}\right) \cap \operatorname{co}\left(A_{2}\right)=\emptyset$ and such that $Q_{1}\left(e \in A_{1}\right)=0$. Let us define $u$ such that $u^{\prime}=\alpha 1_{A_{1}}+1_{A_{2}}$ with $\alpha \geq 0$. We have $E^{Q_{2}}\left[u^{\prime}(e) e\right] E^{Q_{1}}\left[u^{\prime}(e)\right]-E^{Q_{2}}\left[u^{\prime}(e)\right] E^{Q_{1}}\left[e u^{\prime}(e)\right] \geq 0$ or equivalently

$\alpha\left(E^{Q_{2}}\left[e 1_{e \in A_{1}}\right] Q_{1}\left[A_{2}\right]-Q_{2}\left[A_{1}\right] E^{Q_{1}}\left[e 1_{e \in A_{2}}\right]\right)+E^{Q_{2}}\left[e 1_{e \in A_{2}}\right] Q_{1}\left[A_{2}\right]-Q_{2}\left[A_{2}\right] E^{Q_{1}}\left[e 1_{e \in A_{2}}\right] \geq 0$

for all $\alpha \geq 0$. If we assume $Q_{i}\left(e \in A_{j}\right) \neq 0$ for $i \neq j$, we have $\frac{E^{Q_{2}}\left[e 1_{e \in A_{1}}\right]}{Q_{2}\left[e \in A_{1}\right]} \geq$ $\frac{E^{Q_{1}\left[e 1_{e \in A_{2}}\right]}}{Q_{1}\left[e \in A_{2}\right]}$ which leads to $A_{2}<A_{1}$.

Let us now define $x_{1} \equiv \inf \left\{x: Q_{1}(e>x)=0\right\}$ and let $A \subset\left(-\infty, x_{1}-\varepsilon\right)$ for some $\varepsilon>0$ such that $Q_{1}(e \in A)=0$. By construction, $Q_{1}\left(e \in\left[x_{1}-\varepsilon, x_{1}\right]\right) \neq 0$. If we assume $Q_{2}(e \in A) \neq 0$, we have $\left[x_{1}-\varepsilon, x_{1}\right]<A$ and this is impossible. We have then $Q_{2}(e \in A)=0$. Consequently, for all $\varepsilon>0,\left.Q_{2}\right|_{\left\{e \leq x_{1}-\varepsilon\right\}}$ is absolutely continuous with respect to $\left.Q_{1}\right|_{\left\{e \leq x_{1}-\varepsilon\right\}}$, hence $\left.Q_{2}\right|_{\left\{e<x_{1}\right\}}$ is absolutely continuous with respect to $\left.Q_{1}\right|_{\left\{e<x_{1}\right\}}$.

Symmetrically, if we define $x_{2}^{\prime}=\sup \left\{x: Q_{2}(e<x)=0\right\}$, we obtain that $\left.Q_{1}\right|_{\left\{x_{2}^{\prime}<e\right\}}$ is absolutely continuous with respect to $\left.Q_{2}\right|_{\left\{x_{2}^{\prime}<e\right\}}$.

If $x_{2}^{\prime}>x_{1}$, we take $x_{2}=x_{1}$ and $h=0$ and we have $-\infty \leq x_{2} \leq x_{1} \leq \infty$, $Q_{2}\left(e<x_{2}\right)=0, Q_{1}\left(e>x_{1}\right)=0,\left.Q_{2}\right|_{\left\{x_{2} \leq e \leq x_{1}\right\}}$ is absolutely continuous with respect to $\left.Q_{1}\right|_{\left\{x_{2} \leq e \leq x_{1}\right\}}$ and $\frac{\left.d Q_{2}\right|_{\left\{x_{2} \leq e \leq x_{1}\right\}}}{\left.d Q_{1}\right|_{\left\{x_{2} \leq e \leq x_{1}\right\}}}=h(e)$.

If $x_{2}^{\prime} \leq x_{1}$,we take $x_{2}=x_{2}^{\prime}$ and $\left.Q_{2}\right|_{\left\{x_{2} \leq e \leq x_{1}\right\}}$ and $\left.Q_{1}\right|_{\left\{x_{2} \leq e \leq x_{1}\right\}}$ are equivalent. The same argument as in 1 . gives us then the existence of a nondecreasing nonnegative function $h$ such that $\frac{\left.d Q_{2}\right|_{\left\{x_{2}<e<x_{1}\right\}}}{\left.d Q_{1}\right|_{\left\{x_{2}<e<x_{1}\right\}}}=h(e)$.

If $Q_{1}\left(e=x_{1}\right)=0($ resp. $\neq 0)$ and $Q_{2}\left(e=x_{1}\right)=0($ resp. $\neq 0)$ then the previous reasoning can be extended to $\left\{x_{2}<e \leq x_{1}\right\}$. If $Q_{1}\left(e=x_{1}\right)=0$ and $Q_{2}\left(e=x_{1}\right) \neq 0$ then it suffices to take $h\left(x_{1}\right)=\infty$. Finally, it is easy to check that we can not have $Q_{1}\left(e=x_{1}\right) \neq 0$ and $Q_{2}\left(e=x_{1}\right)=0$. The point $x_{2}$ is treated analogously.

In order to establish the converse implication, it suffices to prove, for $A=$ $\left\{x_{2} \leq e \leq x_{1}\right\}$, that $E^{Q_{2}}\left[u^{\prime}(e) e 1_{A}\right] E^{Q_{1}}\left[u^{\prime}(e) 1_{A}\right]-E^{Q_{2}}\left[u^{\prime}(e) 1_{A}\right] E^{Q_{1}}\left[e u^{\prime}(e) 1_{A}\right] \geq$ 0 for all $u$ in $\mathcal{U}_{1}$. Remark that this quantity is not modified if we replace $Q_{1}, Q_{2}$ and $u^{\prime}(e)$ by $\left.Q_{1}\right|_{\left\{x_{2} \leq e \leq x_{1}\right\}},\left.Q_{2}\right|_{\left\{x_{2} \leq e \leq x_{1}\right\}}$ and $u^{\prime}(e) 1_{\left\{x_{2} \leq e \leq x_{1}\right\}}$. The result is then a direct consequence of 1 .

\footnotetext{
${ }^{10}$ If $A$ is a given real subset, we denote by $\operatorname{co}(A)$ the convex hull of $A$.
} 
In fact, if we interpret our result in terms of portfolio dominance, we have established that the MLR property is equivalent to the first order conditions for portfolio dominance for all agents with nondecreasing utility functions.

With the characterization obtained through Proposition 3.1, the $\mathrm{MPR}_{1}$ order is now easily verifiable. If $e$ has a normal distribution $\mathcal{N}\left(\mu_{1}, \sigma_{1}\right)\left(\operatorname{resp} . \mathcal{N}\left(\mu_{2}, \sigma_{2}\right)\right)$ under $Q_{1}$ (resp. under $Q_{2}$ ) then $Q_{1} \succcurlyeq_{M P R_{1}} Q_{2}$ if and only if $\sigma_{1}=\sigma_{2}$ and $\mu_{1} \leq \mu_{2}$. The lognormal case works similarly ${ }^{11}$.

The comonotonicity condition between $\frac{d Q_{2}}{d Q_{1}}$ and $e$ means that " $Q_{1}$ is more pessimistic than $Q_{2}$ " in the sense that it puts more weight when aggregate wealth is low. An $M P R_{1}$ shift in the subjective beliefs can then be interpreted as a pessimistic shift. In particular, in the case of normal distributions, an $M P R_{1}$ shift in the beliefs corresponds to a lower subjective expected value for $e$ with no modification of the risk level. This notion of pessimism is stronger than the one introduced in Abel (2002) since it is immediate and well known that if $F_{2} \succcurlyeq_{M L R}$ $F_{1}$, then $F_{2} \succcurlyeq_{F S D} F_{1}$.

\section{Second-order market-price-of-risk dominance}

Abel (2002) defines doubt as a mean preserving spread of the distribution of growth rates of aggregate consumption. As previously underlined, the common stochastic orders fail to rank demand in portfolio problems, hence it is intuitive that second-order stochastic dominance should not characterize marketprice-of-risk dominance of the second-order. Abel (2002) proves, for power utility functions, and using Taylor series expansions, that doubt reduces the average equity premium. In fact, this result is wrong ${ }^{12}$ as easily seen on the following simple couterexample. Let us consider ${ }^{13} \Omega=\{0.9 ; 1 ; 1.1 ; 2 ; 25 ; 48\}$ with $P=\left(0 ; \frac{1}{2} ; 0 ; 0 ; \frac{1}{2} ; 0\right)$ and $Q=\left(\frac{1}{4} ; 0 ; \frac{1}{4} ; \frac{1}{4} ; 0 ; \frac{1}{4}\right)$. It is easy to see that $Q$ is a mean preserving spread of $P$. If we consider the utility function $u(x)=-\frac{1}{x}$, we obtain $\frac{E^{P}\left[u^{\prime}(e) e\right]}{E^{P}\left[u^{\prime}(e)\right]}=1,04$ and $\frac{E^{Q}\left[u^{\prime}(e) e\right]}{E^{Q}\left[u^{\prime}(e)\right]}=1,10$ which means that the risk premium under

\footnotetext{
${ }^{11}$ As underlined by Landsberger and Meilijson (1990), this order holds for exponential type families (exponential, geometric, Poisson, binomial with the same number of trials, Gamma with the same shape parameter).

${ }^{12}$ Abel's proof is based on second order Taylor expansions and provides an approximate estimation of the risk premia. Focusing on the two first moments is more or less equivalent to restricting ones attention to normal distributions for which all mean-preserving dominance concept coincide with a variance ranking.

${ }^{13}$ The states of the world are indexed by the values taken by $e$.
} 
$Q$ is lower than under $P$.

Our aim is to characterize second order market-price-of-risk dominance. With the same notations as in the previous section ${ }^{14}$, let $X_{1} \sim F_{1}$ and $X_{2} \sim F_{2}$. Then, second-order market-price-of-risk dominance, which is given by $\frac{E^{Q_{2}}\left[u^{\prime}(e) e\right]}{E^{Q_{2}}\left[u^{\prime}(e)\right]} \leq$ $\frac{E^{Q_{1}}\left[u^{\prime}(e) e\right]}{E^{Q_{1}}\left[u^{\prime}(e)\right]}$ for all nondecreasing and concave utility functions $u$ is equivalent to

$$
\frac{E\left[u^{\prime}\left(X_{2}\right) X_{2}\right]}{E\left[u^{\prime}\left(X_{2}\right)\right]} \leq \frac{E\left[u^{\prime}\left(X_{1}\right) X_{1}\right]}{E\left[u^{\prime}\left(X_{1}\right)\right]}
$$

or equivalently

$$
E\left[u^{\prime}\left(X_{1}\right)\left(X_{1}-\rho\right)\right]=0 \Rightarrow E\left[u^{\prime}\left(X_{2}\right)\left(X_{2}-\rho\right)\right] \leq 0 \forall \rho .
$$

As shown by Gollier (1997, Condition (6)), this last condition characterizes Portfolio Dominance, a concept introduced in the context of portfolio problems ${ }^{15}$ by Landsberger and Meilijson (1993) and further studied by Gollier (1997).

Using Proposition 1 in Gollier (1997), we easily deduce that $Q_{1} \succeq_{M P R_{2}} Q_{2}$ if and only if

$$
\forall(x, y) \in \mathbb{R}_{+}^{2} \times \mathbb{R}_{+}^{2}, \quad \frac{\sum_{i=1}^{2} y_{i} E^{Q_{1}}\left[e 1_{e \leq x_{i}}\right]}{\sum_{i=1}^{2} y_{i} E^{Q_{1}}\left[1_{e \leq x_{i}}\right]} \leq \frac{\sum_{i=1}^{2} y_{i} E^{Q_{2}}\left[e 1_{e \leq x_{i}}\right]}{\sum_{i=1}^{2} y_{i} E^{Q_{2}}\left[1_{e \leq x_{i}}\right]} .
$$

Since this condition is not intuitive and is hard to check, we give in the following some simple necessary or sufficient conditions for second-order market-price-of-risk dominance, we relate it to the common first and second stochastic dominances, we interpret our dominance concept in terms of pessimism and doubt and illustrate it by simple examples.

Notice first that, as shown by Landsberger and Meilijson (1993), mean preserving spreads are neither sufficient nor necessary for portfolio dominance, hence Abel's concept of doubt fails to rank market price of risk under subjective beliefs in a general setting.

It is well known that FSD is neither sufficient nor necessary in order to ensure portfolio dominance, and so it is for our concept. In particular, it is immediate

\footnotetext{
${ }^{14}$ We recall that $F_{i}$ denotes the distribution of $e$ under $Q_{i}, i=1,2$.

${ }^{15}$ More precisely, we recall that in the standard portfolio problem, a shift in the distribution of the risky asset is "portfolio dominated" if it reduces the demand for the risky asset for all risk-averse agents, irrespective of the risk free rate. Landsberger and Meilijson (1993) restricted their attention to mean-preserving changes in distribution.
} 
to see that if there exists $\lambda$ such that, for all $x, E^{Q_{1}}\left[e 1_{e \leq x}\right] \leq \lambda E^{Q_{2}}\left[e 1_{e \leq x}\right]$ and $Q_{1}\{e \leq x\} \geq \lambda Q_{2}\{e \leq x\}$, then $Q_{1} \succcurlyeq_{M P R_{2}} Q_{2}$. Figures 3 and 4 provide an example in which the previous conditions are satisfied but not FSD. Moreover, taking $\lambda=1$, these conditions correspond to FSD, i.e. $Q_{1}$ puts more weight on $\{e \leq x\}$ than $Q_{2}$, with the additional condition that on $\{e \leq x\}, Q_{1}$ puts more weight on the low values. This condition seems to be closely related to the comonotonicity condition that characterizes the first-order market-price-ofrisk dominance but it can be shown on simple examples that these two conditions are not equivalent. Figure 5 provides such an example.

The next Proposition shows that all distributions, which are mean-preserving $M P R_{2}$ ordered are also variance ordered.

Proposition 4.1. Let $Q_{1}$ and $Q_{2}$ denote two probability measures on $(\Omega, F)$. If $Q_{1} \succcurlyeq_{M P R_{2}} Q_{2}$, if $E^{Q_{i}}\left[e^{2}\right]<\infty, i=1,2$ and if $E^{Q_{1}}[e]=E^{Q_{2}}[e]$ then $\operatorname{Var}^{Q_{1}}[e] \geq$ $\operatorname{Var}^{Q_{2}}[e]$

Proof. Let us first assume that $e$ has a finite support and let $\bar{e}$ be such that $P\{e \geq \bar{e}\}=0$. Taking $u^{\prime}(x)=(\bar{e}-x) 1_{x \leq \bar{e}}$, we have

$$
\frac{\bar{e} E^{Q_{1}}[e]-E^{Q_{1}}\left[e^{2}\right]}{\bar{e}-E^{Q_{1}}[e]} \leq \frac{\bar{e} E^{Q_{2}}[e]-E^{Q_{2}}\left[e^{2}\right]}{\bar{e}-E^{Q_{2}}[e]} .
$$

Since we assumed $E^{Q_{1}}[e]=E^{Q_{2}}[e]$, we have $E^{Q_{1}}\left[e^{2}\right] \geq E^{Q_{2}}\left[e^{2}\right]$ or equivalently $\operatorname{Var}^{Q_{1}}[e] \geq \operatorname{Var}^{Q_{2}}[e]$. If $e$ does not have a finite support, we still take $u^{\prime}(x)=($ $\bar{e}-x) 1_{x \leq \bar{e}}$ for some $\bar{e}$. Since $E^{Q_{1}}\left[e^{2} 1_{e \leq \bar{e}}\right] \quad$ (resp. $E^{Q_{2}}\left[e^{2} 1_{e \leq \bar{e}}\right]$ ) converges to $E^{Q_{1}}\left[e^{2}\right]$ (resp. $E^{Q_{2}}\left[e^{2}\right]$ ) when $\bar{e}$ goes to infinity, we have for $\varepsilon>0$ given and for $\bar{e}$ sufficiently large

$$
E^{Q_{i}}\left[e^{k}\right]-\bar{e}^{k-2} \varepsilon \leq E^{Q_{i}}\left[e^{k} 1_{e \leq \bar{e}}\right] \leq E^{Q_{i}}\left[e^{k}\right], \quad k=0,1,2, \quad i=1,2
$$

We have then

$$
\frac{\bar{e}\left(E^{Q_{1}}[e]-\frac{1}{\bar{e}} \varepsilon\right)-E^{Q_{1}}\left[e^{2}\right]}{\bar{e}-\left(E^{Q_{1}}[e]-\frac{1}{\bar{e}} \varepsilon\right)} \leq \frac{\bar{e} E^{Q_{2}}[e]-\left(E^{Q_{2}}\left[e^{2}\right]-\varepsilon\right)}{\bar{e}\left(1-\frac{1}{\bar{e}^{2}} \varepsilon\right)-E^{Q_{2}}[e]}
$$

which leads to

$$
\left[\bar{e} E^{Q_{1}}\left[e-\frac{e^{2}}{\bar{e}}\right]-\varepsilon\right]\left[\bar{e}-E^{Q_{1}}[e]-\frac{\varepsilon}{\bar{e}}\right] \leq\left[\bar{e} E^{Q_{1}}\left[e-\frac{e^{2}}{\bar{e}}\right]+\varepsilon\right]\left[\bar{e}-E^{Q_{1}}[e]+\frac{\varepsilon}{\bar{e}}\right] .
$$


Dividing by $\bar{e}$ and taking the limit when $\bar{e}$ goes to infinity and $\varepsilon$ goes to zero we obtain $E^{Q_{1}}\left[e^{2}\right] \geq E^{Q_{2}}\left[e^{2}\right]$ or equivalently $\operatorname{Var}^{Q_{1}}[e] \geq \operatorname{Var}^{Q_{2}}[e]$.

The next proposition provides conditions that ensure second-order marketprice-of-risk dominance. This result is somehow a converse of the result stated in the previous proposition. Indeed, if the probability measures $Q_{1}$ and $Q_{2}$ satisfy these conditions, then $e$ has the same expectation under the two probabilities and has a higher variance under $Q_{1}$.

Proposition 4.2. If the density of $e$ under $Q_{2}$ is symmetric (with respect to $\left.E^{Q_{2}}[e]\right)$ and if the density of $Q_{1}$ with respect to $Q_{2}$ is a function of e, symmetric with respect to $E^{Q_{2}}[e]$, nonincreasing before $E^{Q_{2}}[e]$ and nondecreasing after $E^{Q_{2}}[e]$ then $Q_{1} \succcurlyeq_{M P R_{2}} Q_{2}$.

Proof. Suppose that the density of $Q_{1}$ with respect to $Q_{2}$ can be written in the form $\frac{d Q_{1}}{d Q_{2}}=f\left(e-E^{Q_{2}}[e]\right)$ where $f$ is an even function, increasing on $\mathbb{R}_{+}$. We have

$$
\frac{E^{Q_{1}}\left(e u^{\prime}(e)\right)}{E^{Q_{1}}\left(u^{\prime}(e)\right)}=\frac{E^{Q_{2}}\left[\alpha f(\alpha)\left(u^{\prime}(m+\alpha)-u^{\prime}(m-\alpha)\right) 1_{\alpha \geq 0}\right]}{E^{Q_{2}}\left[f(\alpha)\left(u^{\prime}(m+\alpha)+u^{\prime}(m-\alpha)\right) 1_{\alpha \geq 0}\right]} .
$$

where $m=E^{Q_{2}}[e]$ and $\alpha=e-m$. We want to compare this quantity with

$$
\frac{E^{Q_{2}}\left(e u^{\prime}(e)\right)}{E^{Q_{2}}\left(u^{\prime}(e)\right)}=\frac{E^{Q_{2}}\left[\alpha\left(u^{\prime}(m+\alpha)-u^{\prime}(m-\alpha)\right) 1_{\alpha \geq 0}\right]}{E^{Q_{2}}\left[\left(u^{\prime}(m+\alpha)+u^{\prime}(m-\alpha)\right) 1_{\alpha \geq 0}\right]} .
$$

Letting $g(\alpha)=\alpha\left(u^{\prime}(m-\alpha)-u^{\prime}(m+\alpha)\right)$ and $h(\alpha) \equiv\left(u^{\prime}(m+\alpha)+u^{\prime}(m-\alpha)\right)$, we are led to compare $\frac{E^{Q_{2}}\left[f(\alpha) g(\alpha) 1_{\alpha \geq 0}\right]}{E^{Q_{2}}\left[f(\alpha) h\left(\alpha 1_{\alpha \geq 0}\right]\right.}$ with $\frac{E^{Q_{2}}\left[g(\alpha) 1_{\alpha \geq 0}\right]}{E^{Q_{2}}\left[h(\alpha) 1_{\alpha \geq 0}\right]}$, or equivalently $\frac{E^{Q_{2}}\left[f(\alpha) g(\alpha) 1_{\alpha \geq 0}\right]}{E^{Q_{2}}\left[g(\alpha) 1_{\alpha \geq 0}\right]}$ with $\frac{E^{Q_{2}}\left[f(\alpha) h(\alpha) 1_{\alpha \geq 0}\right]}{E^{Q_{2}}\left[h(\alpha) 1_{\alpha \geq 0}\right]}$. Let us now define the probability measure $P^{g}$ by $\frac{d P^{g}}{d Q_{2}}=$ $\frac{g(\alpha) 1_{\alpha \geq 0}}{E^{Q_{2}}\left[g(\alpha) 1_{\alpha \geq 0}\right]}$. We are led to compare $E^{P^{g}}[f(\alpha)]$ with $E^{P^{g}}\left[f(\alpha) \frac{h}{g}(\alpha)\right] \frac{E^{Q_{2}}\left[g(\alpha) 1_{\alpha \geq 0}\right]}{E^{Q_{2}}\left[h(\alpha) 1_{\alpha \geq 0}\right]}$. The probability measure $Q_{1}$ dominates $Q_{2}$ in the sense of the market price of risk of the second-order if and only if,

$$
E^{P^{g}}[f(\alpha)] \geq E^{P^{g}}\left[f(\alpha) \frac{h}{g}(\alpha)\right] \frac{E^{Q_{2}}\left[g(\alpha) 1_{\alpha \geq 0}\right]}{E^{Q_{2}}\left[h(\alpha) 1_{\alpha \geq 0}\right]} .
$$

It is easy to check that the function $\frac{h}{g}: x \mapsto \frac{\left(u^{\prime}(m+x)+u^{\prime}(m-x)\right)}{x\left(u^{\prime}(m-x)-u^{\prime}(m+x)\right)}$ is decreasing on $\mathbb{R}_{+}$. Since $f$ is increasing, then for any probability measure $Q$, we have 
$\operatorname{cov}^{Q}\left(\frac{h}{g}(\alpha), f(\alpha)\right) \leq 0$, and in particular, $\operatorname{cov}^{P^{g}}\left(\frac{h}{g}(\alpha), f(\alpha)\right) \leq 0$. This implies that

$$
\begin{aligned}
E^{P^{g}}\left[f(\alpha) \frac{h}{g}(\alpha)\right] & \leq E^{P^{g}}[f(\alpha)] E^{P^{g}}\left[\frac{h}{g}(\alpha)\right] \\
& \leq E^{P^{g}}[f(\alpha)] \frac{E^{Q_{2}}\left[h(\alpha) 1_{\alpha \geq 0}\right]}{E^{Q_{2}}\left[g(\alpha) 1_{\alpha \geq 0}\right]}
\end{aligned}
$$

and $E^{P^{g}}[f(\alpha)] \geq E^{P^{g}}\left[f(\alpha) \frac{h}{g}(\alpha)\right] \frac{E^{Q_{2}}\left[g(\alpha) 1_{\alpha \geq 0}\right]}{E^{Q_{2}}\left[h(\alpha) 1_{\alpha \geq 0}\right]}$. This concludes the proof of the Proposition.

The two previous propositions are consistent with an interpretation of an $M P R_{2}$ shift as some form of doubt. However, it is immediate (see e.g. Landsberger and Meilijson, 1993), that our dominance concept implies that for all $x$, $E^{Q_{1}}[e \mid e \leq x] \leq E^{Q_{2}}[e \mid e \leq x]$, which intuitively can be interpreted as some form of pessimism.

As underlined by Landberger and Meilijson (1993), Equal-Mean Normal, Uniform and Shifted Exponential distributions are mean-preserving portfolio ordered (hence $M P R_{2}$ ordered) by their variances. Moreover, we have the following result.

Example 4.3. Let us assume that $X_{1} \sim \mathcal{N}\left(\mu_{1}, \sigma_{1}\right)$ and $X_{2} \sim \mathcal{N}\left(\mu_{2}, \sigma_{2}\right)$. We have $Q_{1} \succcurlyeq_{M P R_{2}} Q_{2}$ if and only if $\mu_{1} \leq \mu_{2}$ and $\sigma_{1} \geq \sigma_{2}$.

Proof. 1. If $\mu_{1}=\mu_{2}$ and $\sigma_{1} \geq \sigma_{2}$ then the assumptions of Proposition 4.2 are satisfied and consequently $Q_{1} \succcurlyeq_{M P R_{2}} Q_{2}$. If $\mu_{1} \leq \mu_{2}$ and $\sigma_{1}=\sigma_{2}$, we have already seen that $Q_{1} \succcurlyeq_{M P R_{1}} Q_{2}$ and then $Q_{1} \succcurlyeq_{M P R_{2}} Q_{2}$. If $\mu_{1} \leq \mu_{2}$ and $\sigma_{1} \geq \sigma_{2}$, the result is obtained by transitivity. 2. Conversely, suppose that $Q_{1} \succcurlyeq_{M P R_{2}} Q_{2}$. It suffices to consider the exponential utility function to obtain that $\mu_{1}-a \sigma_{1}^{2} \leq \mu_{2}-a \sigma_{2}^{2}$, for all $a \in(0,+\infty)$, where $a$ is the risk aversion parameter. The result easily follows.

Note that in the lognormal case, we also have that if $Q_{1} \succcurlyeq_{M P R_{2}} Q_{2}$, then $\mu_{1} \leq \mu_{2}$ and $\sigma_{1} \geq \sigma_{2}$ (it suffices to consider power utility functions).

\section{References}

[1] Abel, Andrew, 2002. An exploration of the effects of pessimism and doubt on asset returns. Journal of Economic Dynamics and Control, 26, 1075-1092. 
[2] Cecchetti, S., Lam, P. and N. Mark, 2000. Asset pricing with distorded beliefs: are equity returns too good to be true? American Economic Review, 90, 787805 .

[3] Fishburn, P., and B. Porter, 1976. Optimal portfolios with one safe and one risky asset: Effects of changes in rate of return and risk. Management Science, $22,1064-1073$.

[4] Giordani, P. and P. Söderlind, 2003. Is there evidence of pessimism and doubt in subjective distribution? A comment on Abel. CEPR Discussion Paper.

[5] Gollier, C., 1995. The comparative statics of changes in risk revisited. Journal of Economic Theory, 66, 522-535.

[6] Gollier, C., 1997. A note on portfolio dominance. Review of Economic Studies, 64, 147-150.

[7] Gollier, C. and H. Schlesinger, 2002. Changes in risk and asset prices, Journal of Monetary Economics, 49, 747-760.

[8] Hadar, J. and T. Seo, 1990. The effects of shift in a return distribution on optimal portfolios. International Economic Review, 31, 721-736.

[9] Kocherlakota, N. R., 1996. The Equity Premium: It's Still a Puzzle. Journal of Economic Literature, 34, 42-71.

[10] Landsberger, M., and I. Meilijson, 1990. Demand for risky financial assets: A portfolio analysis. Journal of Economic Theory, 50, 204-213

[11] Landsberger, M., and I. Meilijson, 1993. Mean-preserving portfolio dominance. Review of Economic Studies, 60, 479-485

[12] Mehra, R., and E. Prescott, 1985. The Equity Premium: A Puzzle. Journal of Monetary Economics, 15, 145-162.

[13] Meyer, J. and M. Ormiston, 1983. The comparative statics of cumulative distribution function changes for the class of risk averse agents. Journal of Economic Theory, 31, 151-169.

[14] Ormiston, M.B. and E. Schlee, 1993. Comparative statics under uncertainty for a class of economic agents. Journal of Economic Theory, 61, 412-422. 
[15] Rothschild, M. and J.E. Stiglitz, 1970. Increasing risk : I. A definition. Journal of Economic Theory, 2, 225-243

[16] Rothschild, M. and J.E. Stiglitz, 1970. Increasing risk : II. Its economic consequences. Journal of Economic Theory, 3, 66-84 


\section{FIGURES}

Figure 1

This figure represents the distribution functions of $Q$ (dashed line) and $P$ (solid line).

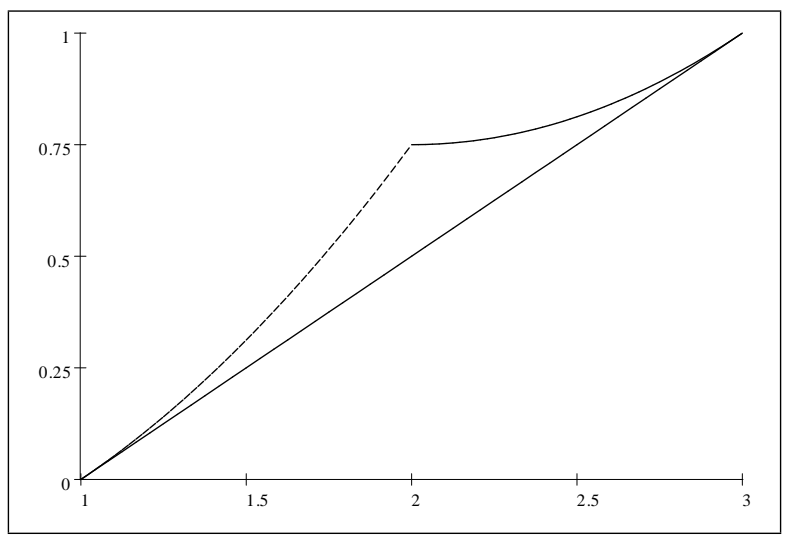

Figure 2

This figure represents the density of $P_{u}$ (solid line) as well as the density of $Q$ with respect to $P$.

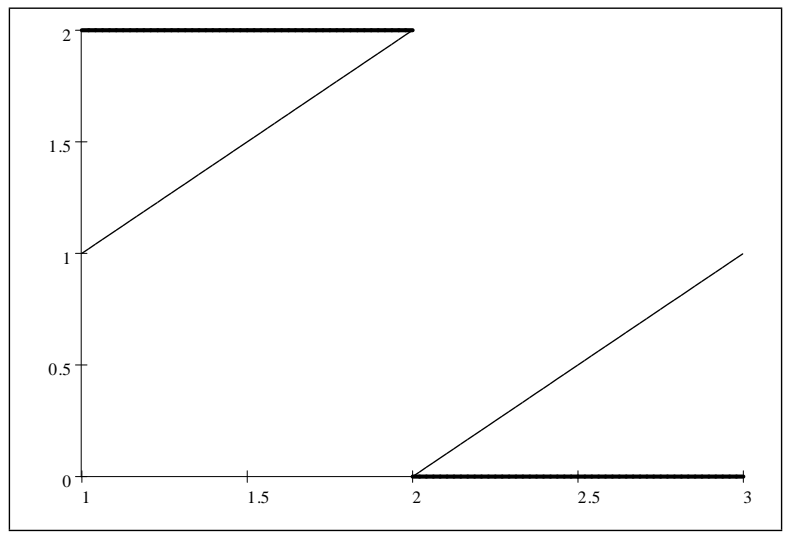




\section{Figure $3 \& 4$}

These figures represent a situation where for some $\lambda, E^{Q_{1}}\left[e 1_{e \leq x}\right] \leq \lambda E^{Q_{2}}\left[e 1_{e \leq x}\right]$ and $Q_{1}\{e \leq x\} \geq \lambda Q_{2}\{e \leq x\}$ but without the first-order stochastic dominance. More precisely, we have $e=(0,1,2), Q_{1}=\left(\frac{1}{3}, \frac{1}{3}, \frac{1}{3}\right)$ and $Q_{2}=\left(\frac{1}{8}, \frac{5}{8}, \frac{2}{8}\right)$. In the first figure, the solid (resp. dashed) thin line represents the distribution function of $Q_{2}$ (resp. $Q_{1}$ ). The solid (resp. dashed) thick line represents the function $E^{Q_{1}}\left[e 1_{e \leq x}\right]$ (resp. $E^{Q_{2}}\left[e 1_{e \leq x}\right]$ ). We clearly do not have first stochastic dominance. In the second figure, the dashed lines are the same as in the previous one and the solid lines correspond to $\lambda Q_{1}[e \leq x]$ and $\lambda E^{Q_{1}}\left[e 1_{e \leq x}\right]$ with $\lambda=\frac{16}{18}$.
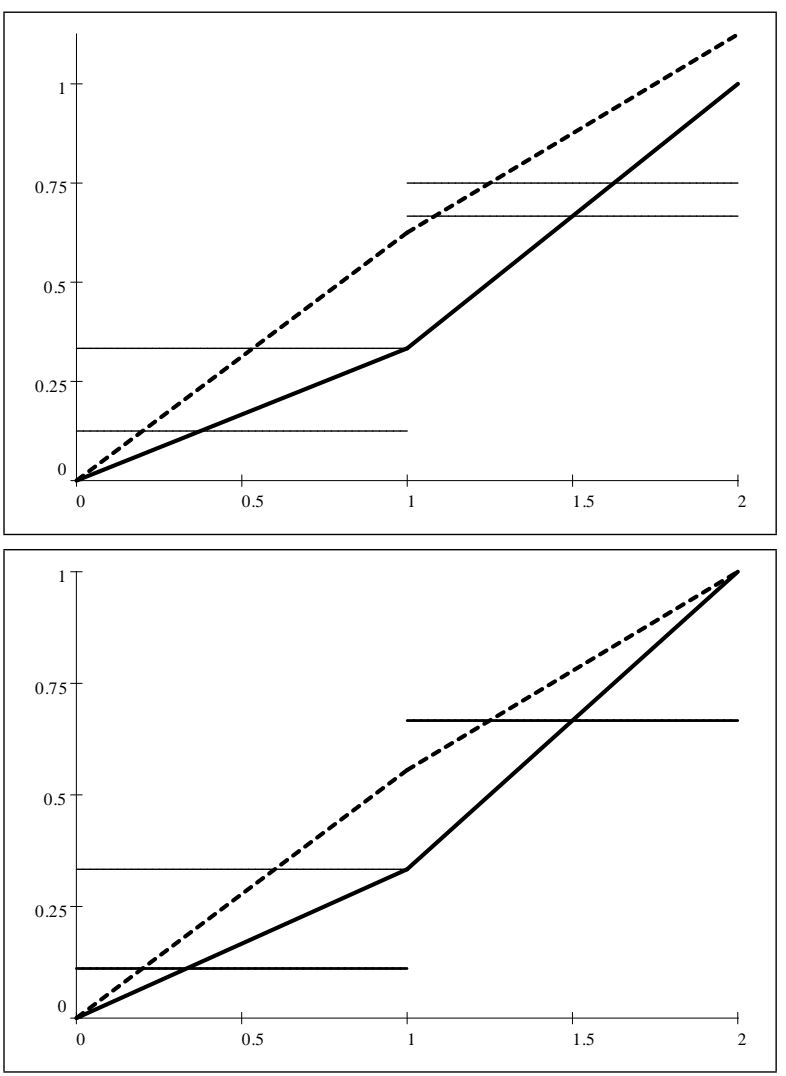


\section{Figure 5}

This figure represents a situation where $E^{Q_{1}}\left[e 1_{e \leq x}\right] \leq E^{Q_{2}}\left[e 1_{e \leq x}\right]$ and $Q_{1}\{e \leq x\} \geq$ $Q_{2}\{e \leq x\}$ but where the comonotonicity condition is not satisfied. More precisely, we have $e=(0,1,2), Q_{1}=\left(\frac{1}{3}, \frac{1}{3}, \frac{1}{3}\right)$ and $Q_{2}=\left(\frac{1}{8}, \frac{4}{8}, \frac{3}{8}\right)$. The solid (resp. dashed) thin line represents the distribution function of $Q_{2}$ (resp. $Q_{1}$ ). The solid (resp. dashed) thick line represents the function $E^{Q_{1}}\left[e 1_{e \leq x}\right]$ (resp. $E^{Q_{2}}\left[e 1_{e \leq x}\right]$ ). The thin (resp. thick) dashed line is clearly above (resp. below) the solid one. The dot-dash line represents $\frac{d Q_{1}}{d Q_{2}}$ and is clearly nonmonotone.

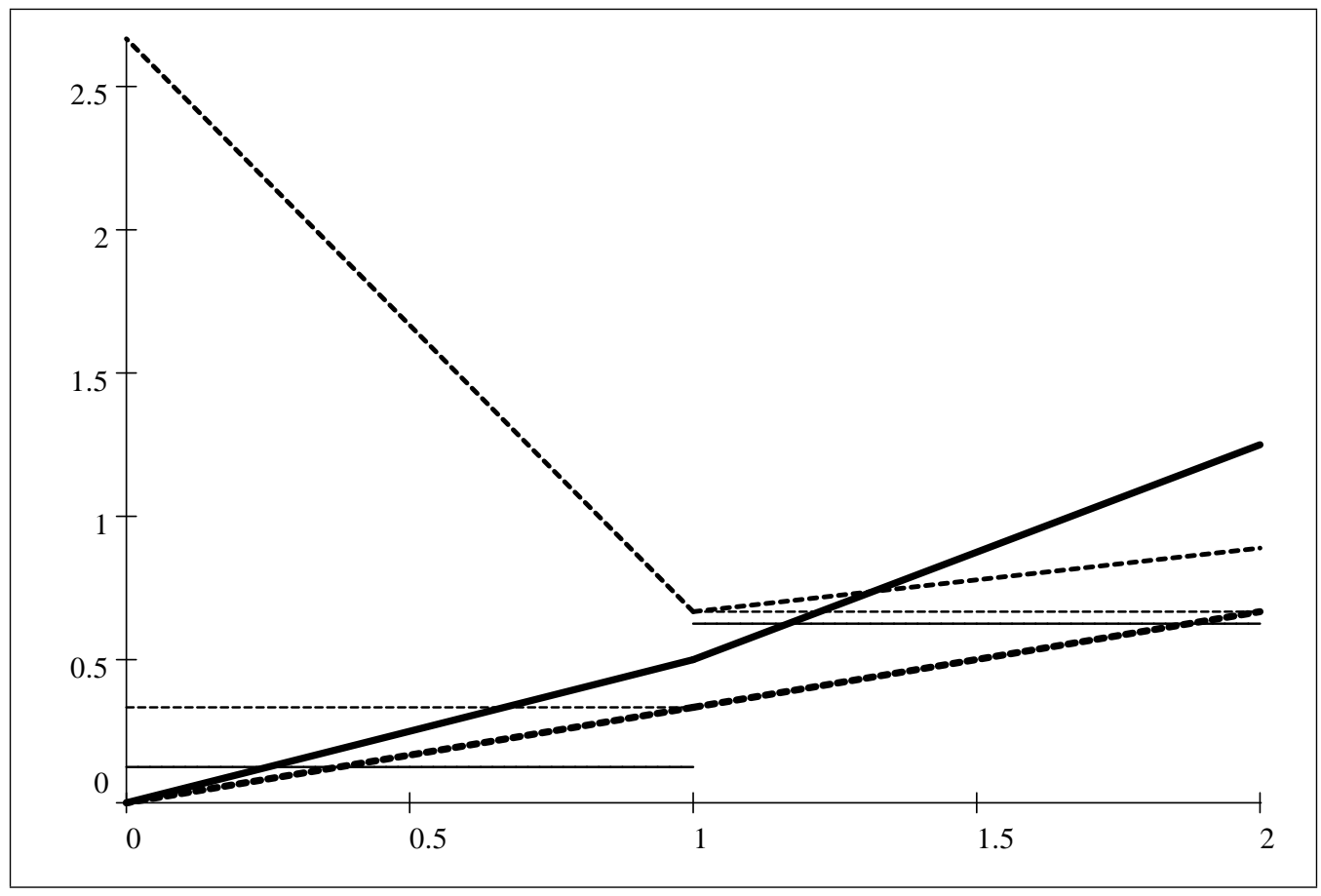

